\title{
QOS-BASED PERFORMANCE EVALUATION OF CHANNEL-AWARE/QOS-AWARE SCHEDULING ALGORITHMS FOR VIDEO- APPLICATIONS OVER LTE/LTE-A
}

\author{
Najem N. Sirhan, Gregory L. Heileman, Christopher C. Lamb, Ricardo \\ Piro-Rael \\ Electrical and Computer Engineering Department, \\ University of New Mexico, Albuquerque, New Mexico, USA \\ najem83@unm. edu heileman@ece.unm.edu cclamb@unm.edu \\ fdisk122r@gmail.com
}

\begin{abstract}
Long Term Evolution (LTE) is defined by the Third Generation Partnership Project (3GPP) standards as Release 8/9. The LTE supports at max $20 \mathrm{MHz}$ channel bandwidth for a carrier. The number of LTE users and their applications are increasing, which increases the demand on the system BW. A new feature of the LTE-Advanced (LTE-A) which is defined in the 3GPP standards as Release 10/11 is called Carrier Aggregation (CA), this feature allows the network to aggregate more carriers in-order to provide a higher bandwidth. Carrier Aggregation has three main cases: Intra-band contiguous, Intra-band non-contiguous, Inter-band contiguous. The main contribution of this paper was in implementing the Intra-band contiguous case by modifying the LTE-Sim-5, then evaluating the Quality of Service $(Q o S)$ performance of the Modified Largest Weighted Delay First (MLWDF), the Exponential Rule (Exp-Rule), and the Logarithmic Rule (Log-Rule) scheduling algorithms over LTE/LTE-A in the Down-Link direction. The QoS performance evaluation is based on the system's average throughput, Packet Loss Rate (PLR), average packet delay, and fairness among users. Simulation results show that the use of CA improved the system's average throughput, and almost doubled the system's maximum throughput. It reduced the PLR values almost by a half. It also reduced the average packet delay by 20-40\\% that varied according to the video bit-rate and the number of users. The fairness indicator was improved with the use of CA by a factor of 10-20\%.
\end{abstract}

\section{KEYWORDS}

Long Term Evolution (LTE), LTE-Advanced (LTE-A), Carrier Aggregation (CA), Quality of Service (QoS), downlink scheduling.

\section{INTRODUCTION}

The LTE was introduced as an evolution to the Universal Mobile Telecommunication Systems (UMTS) to provide cellular network users with high data rates in both the uplink and downlink direction, decreased latency, and good spectrum utilization [1]. The spectrum utilization could be achieved by the use of the right scheduling algorithm that meets with the environment's conditions and the users' requirements demands. There are many scheduling algorithms that exist

Jan Zizka et al. (Eds) : DBDM, CICS, CCNET, CSIP - 2015

pp. 49-65, 2015. (C) CS \& IT-CSCP 2015

DOI : $10.5121 /$ csit.2015.50705 
in the literature that are used in the LTE scheduling process. These algorithms can be classified in five main groups: channel-unaware, channel-aware/QoS-unaware, channel-aware/QoS, semipersistent for VoIP support, and energy-aware [2]. When the number of users and their applications increases, such as video-streaming and video-conferencing, this requires higher data rates and decreased latency, which declines the service that the LTE provides to its users. This challenge of providing a reliable service up to the users' requirements demands can not be solved entirely by choosing the right scheduling algorithm, because the performance of these scheduling algorithms is bounded by the existing LTE capabilities, such as the system's bandwidth. The LTE supports at max $20 \mathrm{MHz}$ channel bandwidth. However, the LTE-A can support more channel bandwidth according to the release as specified in the 3GPP's technical specifications. In Release 10 (R10), the maximum aggregated bandwidth is $40 \mathrm{MHz}$. And it is also $40 \mathrm{MHz}$ in Release 11 (R11), but with much more CA configurations [3]. This was the motivation to evaluate the QoS performance of three Channel-aware/QoS-aware scheduling algorithms for video-applications over the LTE Release 8/9 and LTE-A Release 10/11. The LTE-Sim-5 can only simulate the LTE network without the use of CA, so the performance of these algorithms over LTE-A could not be evaluated without making these modifications to the LTE-Sim-5, which motivated us to modify it in order to make these evaluations.

The structure of this paper is as follows: in section 2, we explained the LTE network architecture. In section 3, we explained the LTE radio spectrum. In section 4, we explained the carrier aggregation. In section 5, we explained the general model of LTE scheduling. In section 6, we explained the LTE scheduling algorithms which we evaluated in this paper. In section 7, we explained the simulation environment and listed its parameters. In section 8, we used the simulation results to measure the QoS parameters which we displayed in line charts and then analysed. In the last section, we provided a concluding remarks.

\section{LTE NETWORK ARCHITECTURE}

The LTE network architecture can be divided into two main parts: the Radio Access Network (RAN), and the Evolved Packet Core (EPC) as in Figure 1. The RAN consists of an Evolved NodeB (eNodeB) and User Equipment (UE). The eNodeB is the connection point for the UE with the core network. It hosts the PHYsical (PHY), Medium Access Control (MAC), Radio Link Control (RLC), and Packet Data Control Protocol (PDCP) layers that include the functionality of user-plane header-compression and encryption. It also offers Radio Resource Control (RRC) functionality that corresponds to the control plane. Scheduling, admission control, and radio resource management are also performed in the eNodeB. The EPC part consists of five main components: the Policy Control and Charging Rules Function (PCRF), the Home Subscriber Server (HSS), the PDN-Gateway (P-GW), the Serving Gateway (S-GW), and the Mobility Management Entity (MME). The PCRF is a logical node responsible for policy control decisionmaking, and controlling the flow-based charging functionalities in the Policy Control Enforcement Function (PCEF) that is being hosted at the P-GW. It also decides how a certain data flow will be treated in the PCEF by providing the QoS authorization, QoS class identification, and determine the bit rates in accordance with the user's subscription profile. The HSS is the database of the LTE network, it contains all the users' subscription QoS profile, information about the Packet Data Networks (PDNs) the user can connect to, dynamic information that relates the identity of the MME to which the user is currently attached or registered to, and it may also integrate the Authentication Center $(\mathrm{AuC})$ that generates the vectors for authentication and security keys. The P-GW is the gateway which is responsible for QoS enforcement for Guaranteed Bit Rate (GBR) bearers, flow-based charging according to rules from the PCRF, and the allocation of IP addresses to users. In addition it filters user's IP packets into different QoSbased bearers based on Traffic Flow Templates (TFTs). It also serves as the mobility anchor for inter-working with non-3GPP networks such as WiMAX and WiFi. The S-GW is the gateway 
that serves as the local mobility anchor for the data bearers while users are moving between eNodeBs, in which all their IP packets are transferred through it. It temporarily buffers user's downlink data when it is in the idle state, while the MME initiates paging of the UE to reestablish the bearers. It performs administrative functions in the visited network such as collecting information for charging and legal interception. It also serves as the mobility anchor for inter-working with 3GPP networks such as General Packet Radio Service (GPRS) and Universal Mobile Telecommunication Systems (UMTS). The MME is the main node in the EPC, it manages the Authentication and Security, and the subscription profile and service connectivity of users. It is responsible for all the mobility management tasks such as inter eNodeBs handovers, inter MMEs handovers, and keeping track of the location of all users [4].
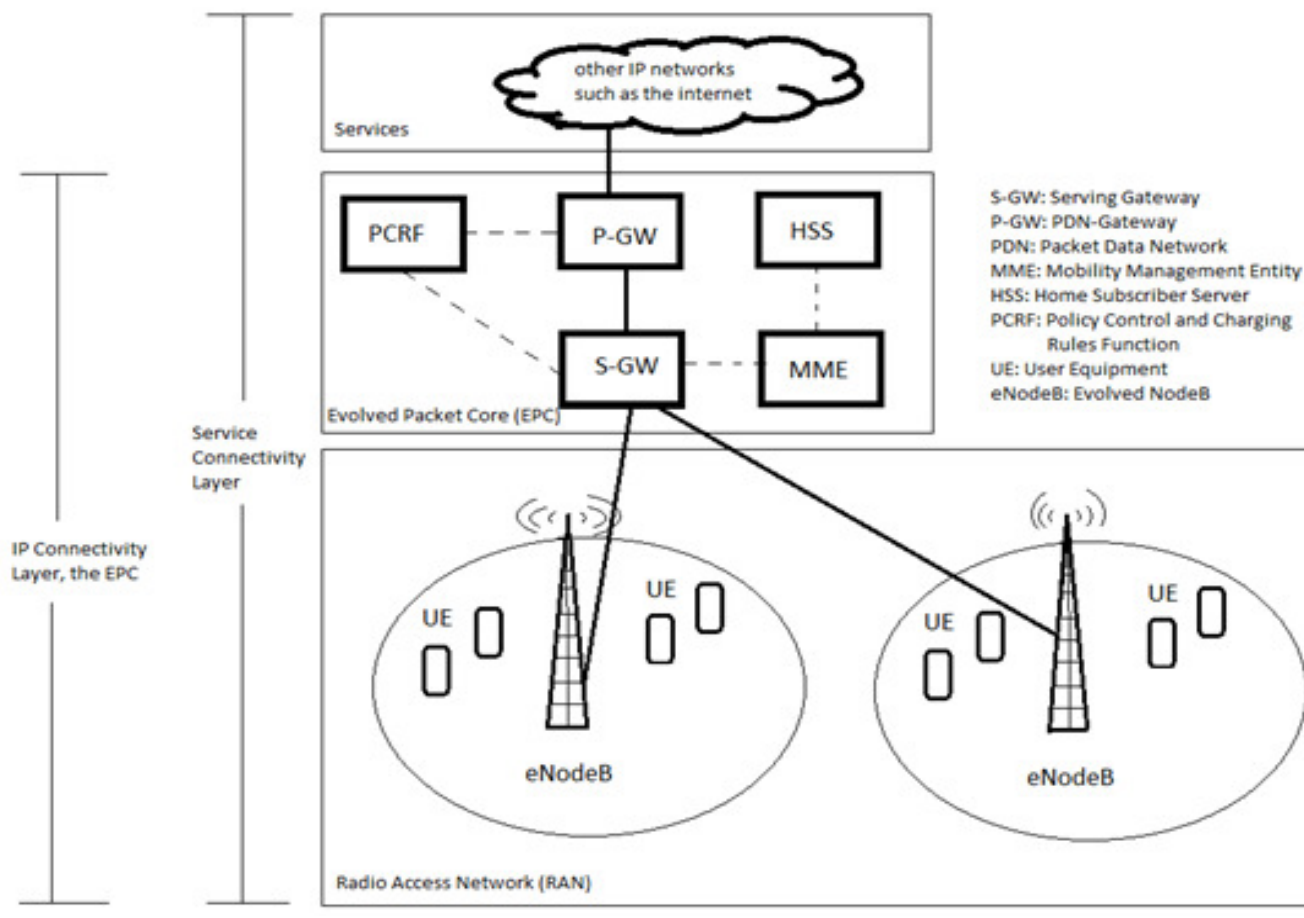

Figure 1. LTE network architecture

\section{LTE RADIO SPECTRUM}

The LTE radio spectrum can be represented in a two dimensional array: one dimension exists in the time domain, the other one in the frequency domain as shown in Figure 2. In the time domain, LTE transmissions are organized into radio frames of length $10 \mathrm{~ms}$, each frame is equally divided into 10 sub-frames " $1 \mathrm{~ms}$ each". This sub-frame is the minimum scheduling unit in LTE, each sub-frame consists of two equal time slots " $0.5 \mathrm{~ms}$ each". There are two types of time slots, one is the normal cyclic prefix which is combined of 7-OFDM symbols, the other is the extended cyclic prefix which is combined of 6-OFDM symbols. In LTE, each downlink sub-frame is divided into two regions: the first one is the control region which consists of two or three OFDM symbols, the remaining part is used for data as shown in figure 3 [5].

In the frequency domain, the bandwidth is divided into sub-carriers, each sub-carrier has a width of $15 \mathrm{KHz}$, and it is occupied by one OFDM symbol, which is the smallest physical resource in LTE. It is called a resource element, as shown in Figure 3. A group of resource elements form a resource block which is extended for a one slot time, consequently the resource block will have a 
width of $180 \mathrm{KHz}$ " $12 * 15 \mathrm{KHz}$ ", and it is the minimum scheduling unit for LTE users. Each subcarrier is being modulated using either Quadrature Phase-Shift Keying (QPSK), 16-Quadrature Amplitude Modulation (16-QAM), or 64-QAM. The number of bits each sub-carrier can occupy at a period of one OFDM symbol time depends on the modulation type [6].

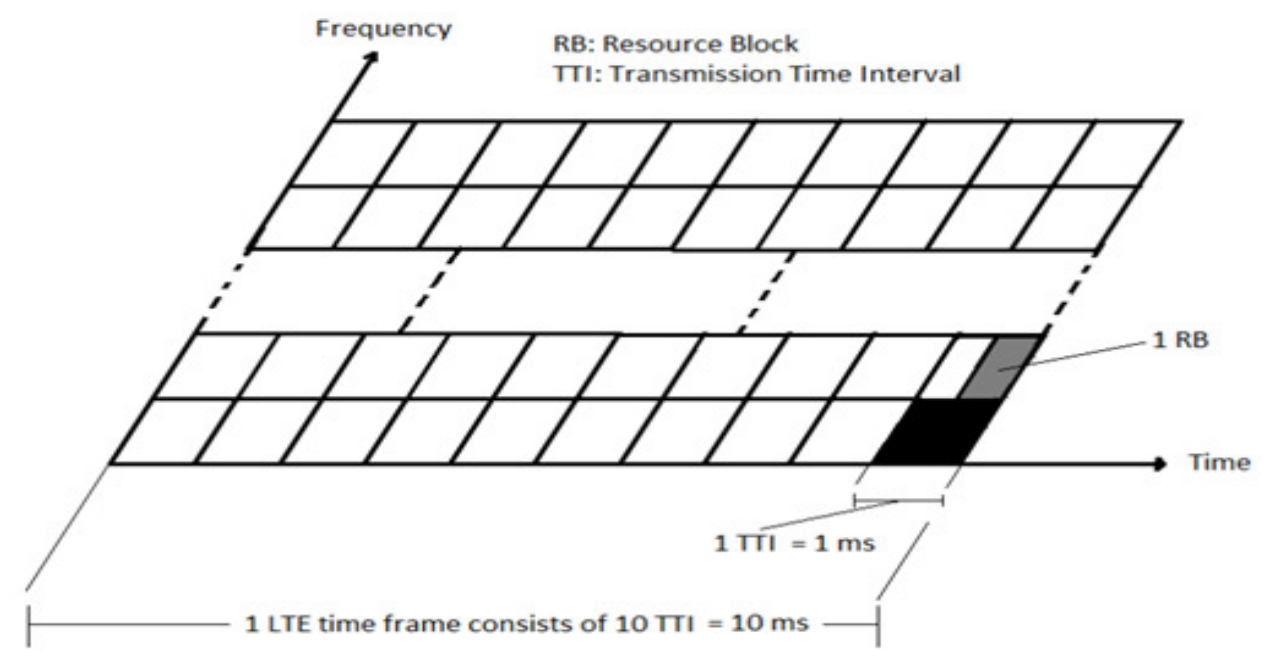

Figure 2. LTE radio spectrum

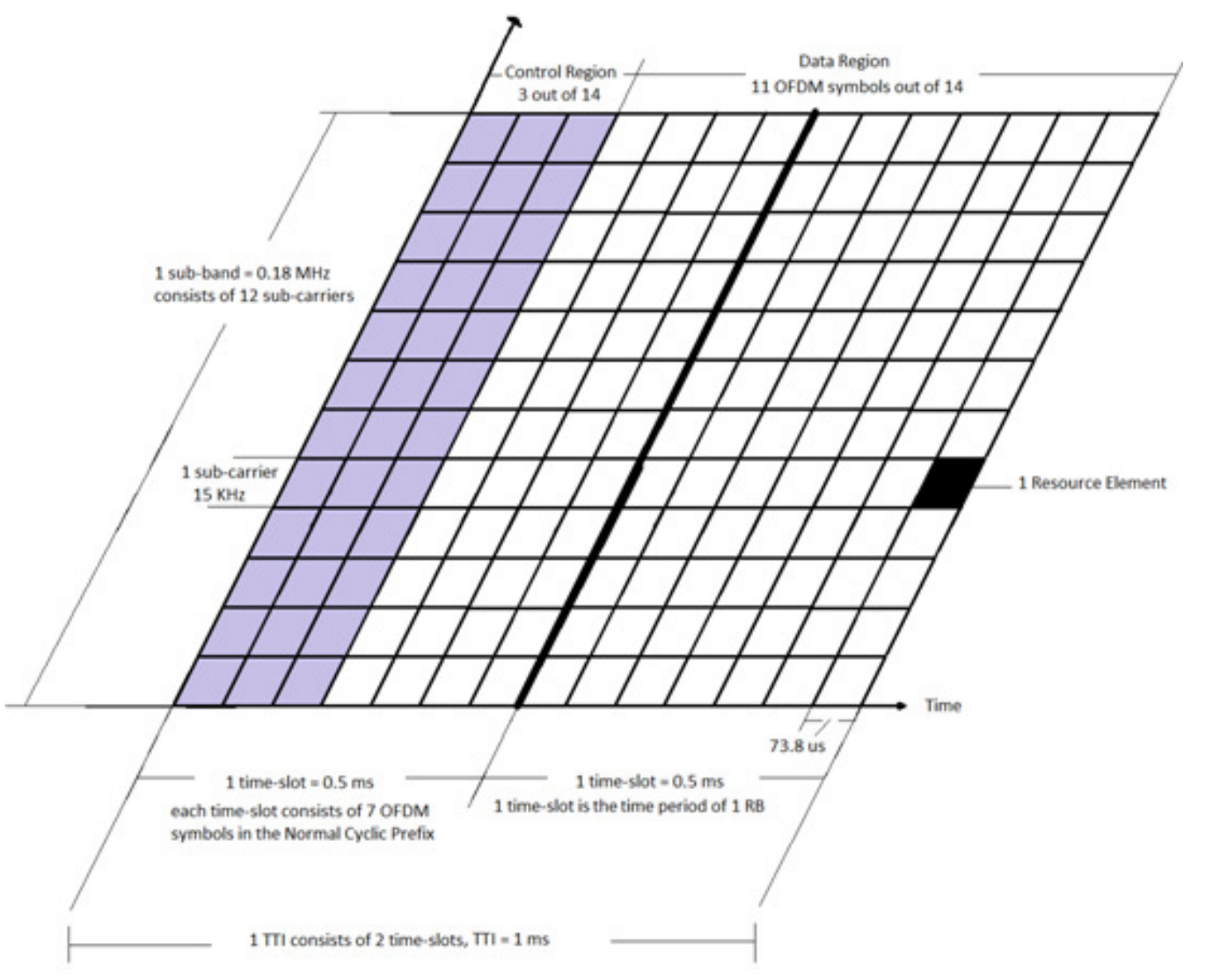

Figure 3. The smallest scheduling LTE unit in the time-frequency domain 


\section{CARrier Aggregation}

The 3GPP Release 8/9 supports at max $20 \mathrm{MHz}$ channel bandwidth for a carrier. The issue of supporting more bandwidth for a carrier seems to be a straight forward solution to support more data rate. Hence the concept of carrier aggregation was introduced where multiple carriers of 20 $\mathrm{MHz}$ (or less) would be aggregated for the same UE. Figure 4 shows the principle of carrier aggregation [7].

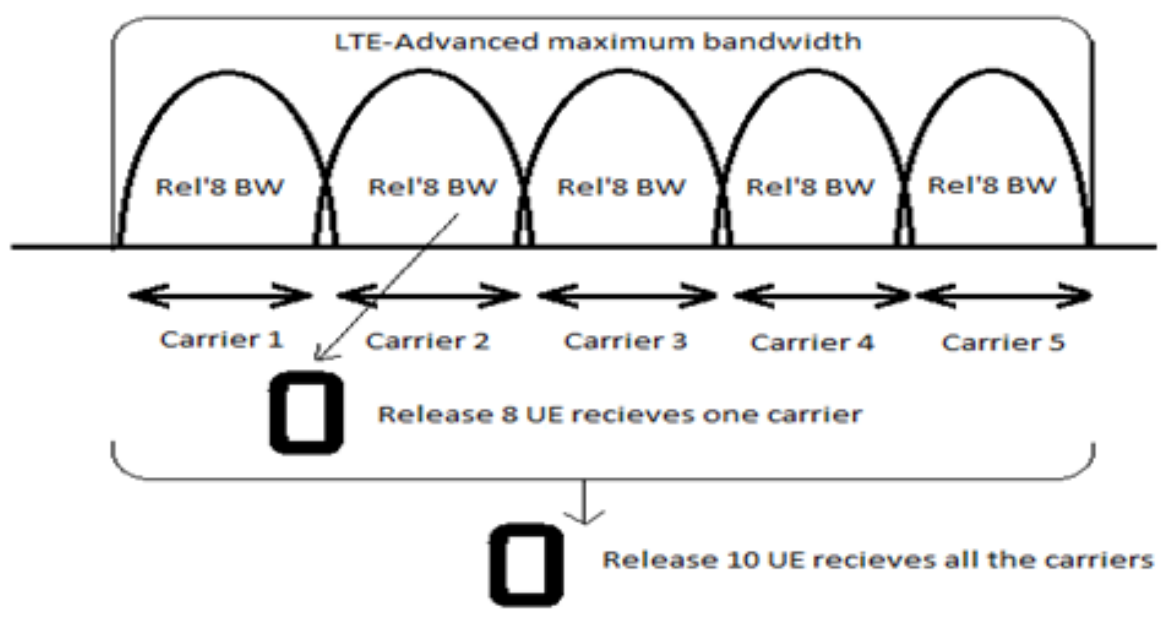

Figure 4. The principle of carrier aggregation

Carrier aggregation in the downlink and the uplink are entirely independent as long as the number of uplink carriers cannot exceed the number of downlink carriers. Each aggregated carrier is called as Component Carrier (CC). 3GPP defined three types of allocation that meets different operator's spectrum scenarios: Intra-band continuous, Intra and Inter-band non-continuous as Figure 5 shows [8].

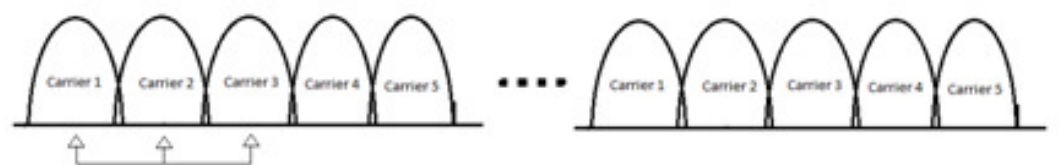

CA cue a: intra and contipuose accomien 1,2 and 3 tron
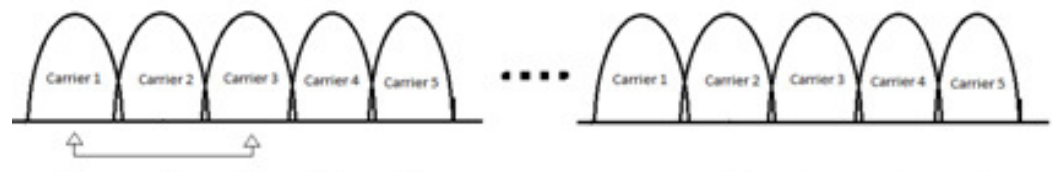
CA case bi intra- and Non-Conbiguoen ex. carress 1 ind 3 trom kind 1 are sened together

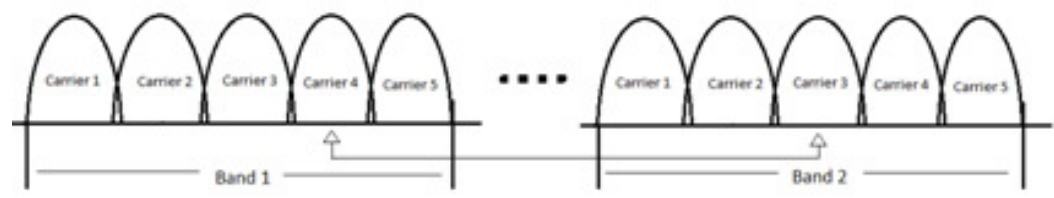

CA cave Cimter - and non-comivuobe trom kund 2 we sumed together

Figure 5. Carrier aggregation cases

\section{LTE SCHEDULING}

Packet schedulers that allocate radio resources to users' packets are deployed by the MAC layer which is hosted at the eNodeB, while users' applications and connections are scheduled by the 
application layer. For allocating the resources to users, a comparison has to be made based on a previously defined metric. This metric could be seen as the priority of each user for a specific resource block. This metric comparison is done every Transmission Time Interval (TTI) in order to perform the allocation decision which is sent to the users over the PDCCH. One of the main characteristics of the Physical Downlink Shared Channel (PDSCH) is to be shared among users, which means that on every TTI which equals $1 \mathrm{~ms}$, portions of the radio spectrum should be distributed among users. The user with the highest metric will be allocated this specific resource block. The Physical Downlink Control Channel (PDCCH) contains the Downlink Control Information (DCI) messages that informs the users about resource blocks allocated for data transmission on the PDSCH in the downlink direction, and about the radio resource that were allocated to their data transmission on the Physical Uplink Control Channel (PUSCH) in the uplink direction [2].

The general model of how a downlink packet scheduler interacts with users is shown in Figure 6 . In [2], they have divided the whole scheduling process in a sequence of operations that are repeated every TTI. First, the UE decodes the reference signal and computes the Channel Quality Indicator (CQI) then sends it back to the eNodB. Second, the CQI information is being used by the eNodeB for making the allocation decisions and filling up a RB allocating mask. Third, the Adaptive Modulation and Coding (AMC) module selects the best Modulation and Coding Scheme (MCS) that should be used for the data which will be transmitted by the scheduled users. Fourth, all the above information are sent to the UEs on the PDCCH. Finally, each UE reads the $\mathrm{PDCCH}$ and accesses to the proper PDSCH if it has been scheduled. This model is slightly different in the case of uplink because the eNodeB does not require extra information about the uplink channel quality.

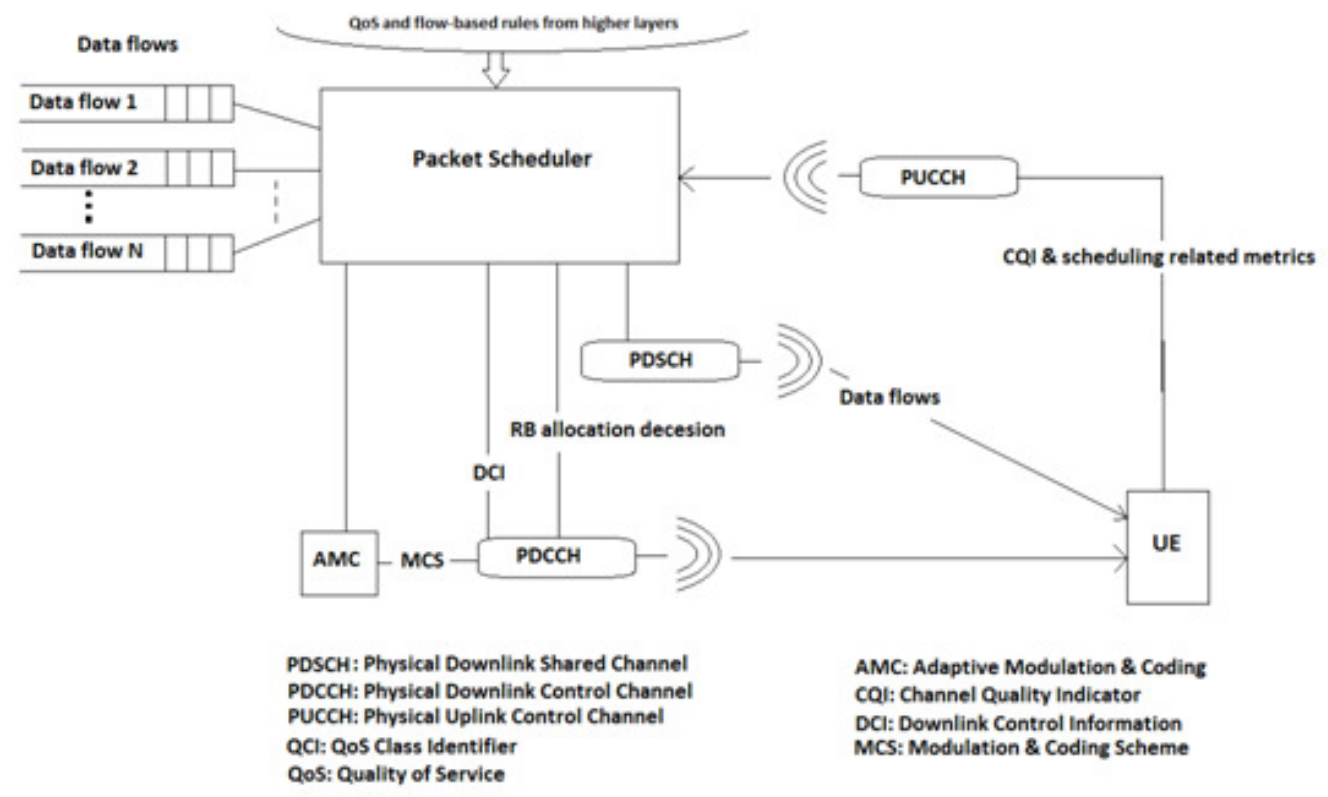

Figure 6. LTE scheduling general model

\section{LTE SCHEDULING ALGORITHMS}

The LTE scheduling algorithms that were studied in this paper are: the Modified Largest Weighted Delay First (MLWDF), the Logarithmic Rule Algorithm (Log-Rule), and the Exponential Rule Algorithm (Exp-Rule). In all these algorithms the Proportional Fairness (PF) scheduler is used in-order to achieve channel awareness, which makes a trade-off between users' 
fairness and spectrum efficiency [2]. It schedule users in a fair way by taking into account both the experienced channel state and the past data rate when assigning radio resources. It aims to

$$
k=\operatorname{argmax} \frac{r_{i}(t)}{R_{i}(t)}
$$

obtain satisfying throughput and at the same time, guarantee fairness among flows. The equation that users are selected based on the following formula [9]:

Where $r_{i}(t)$ is the achievable data rate according to the instantaneous channel quality of user $i$ at $t$ th TTI, and $R_{i}(t)$ is the average data rate of user $i$ over a time window, and it is calculated based on the following formula [9]:

$$
R_{i}(t)=(1-\beta) * R_{i}(t-1)+\beta * r_{i}(t-1)
$$

Where $\beta$ is a variable ranging from 0 to 1 .

\subsection{Modified Largest Weighted Delay First (MLWDF)}

The MLWDF scheduling algorithm is designed to support multiple real time data users by taking into account their different QoS requirements. For example, in the case of video services, the instantaneous channel variations and delays are taken into account. It tries to balance the weighted delays of packets and to utilize the knowledge about the channel state efficiently. It chooses user $j$ at time $t$ based on the following formula [10]:

$$
j=\max _{i} \alpha_{i} \frac{\mu_{i}(t)}{\bar{\mu}} W_{i}(t)
$$

Where $\mu_{i}(t)$ is the data rate corresponding to user $i$ 's channel state at time $t, \mu_{i}(t)$ is the mean data rate supported by the channel, $W i(t)$ is the HOL packet delay and $\alpha_{i}>0, i=1, \ldots, N$ are weights that represent the required level of QoS.

The MLWDF's delay is bounded by the Largest Weighted Delay First (LWDF) scheduler. The LWDF metric is based on the system parameter, representing the acceptable probability for the $i$ th user, in which a packet is dropped due to deadline expiration, and this metric is calculated based on the following [2]:

$$
\begin{gathered}
m_{i, k}^{L W D F}=\alpha_{i} \cdot D_{H O L, i} \\
\alpha_{i}=-\frac{\log \delta_{i}}{\tau_{i}}
\end{gathered}
$$

Where $\alpha_{\mathrm{i}}$ is calculated based on the following formula:

The MLWDF is also expressed in terms of the PF scheduler as in the following formula:

$$
m_{i, k}^{M L W D F}=\alpha_{i} D_{H O L, i} \cdot m_{i, k}^{P F}=\alpha_{i} D_{H O L, i} \cdot \frac{d_{k}^{i}(t)}{\bar{R}^{i}(t-1)}
$$

\subsection{Logarithmic Rule Algorithm (LOG-Rule)}

The delay of this scheduling algorithm is bounded by the following logarithmic formula [2]: 


$$
m_{i, k}^{\text {LOGrule }}=b_{i} \log \left(c+\alpha_{i} D_{H O L, i}\right) \cdot \Gamma_{k}^{i}
$$

Where $\alpha_{i}, b_{i}, c$ are tunable parameters, and the spectral efficiency for the $i$-th user on the $k$-th subchannel is represented by:

$\Gamma_{k}^{i}$

\subsection{Exponential Rule Algorithm (Exp-Rule)}

The delay of this scheduling algorithm is bounded by the following Exponential formula [2]:

$$
m_{i, k}^{E X P r u l e}=b_{i} \exp \left(\frac{\alpha_{i} D_{H O L, i}}{c+\sqrt{\left(1 / N_{r t}\right) \sum_{j} D_{H O L, j}}}\right) \cdot \Gamma_{k}^{i}
$$

\section{SIMULATION ENVIRONMENT}

The simulation environment consists of one Macro cell that is served by one transmitter "eNodeB". The bandwidth was varied from $20 \mathrm{MHz}$ "to represent the LTE maximum bandwidth the case in which CA was not used" to $40 \mathrm{MHz}$ "to represent the LTE-A bandwidth - the case in which the CA was used", the number of users was varied from 15, 30, 60, 90, 120, 150, 180, 210 and the video bit-rate was varied from $128 \mathrm{Kbps}, 242 \mathrm{Kbps}$, and $440 \mathrm{Kbps}$. Detailed parameters of this simulation are listed in Table 1.

Table 1. Simulation Parameters.

\begin{tabular}{|l|l|}
\hline Parameter & Value \\
\hline Simulator & LTE-Sim-5 \\
\hline Simulation time & $20 \mathrm{sec}$ \\
\hline Scheduling Algorithms & Exp-Rule, Log-Rule, MLWDF \\
\hline Network Layout & 1 Macro cell "Urban environment" \\
\hline Transmitter & 1 eNodeB \\
\hline Cell Radius & $1 \mathrm{Km}$ \\
\hline Carrier frequency & $2120,2130 \mathrm{MHz}$ \\
\hline Bandwidth & $2130-2110=20,2150-2110=40 \mathrm{MHz}$ \\
\hline Carrier Aggregation case & Inter-band contiguous \\
\hline Frame structure & FDD \\
\hline Number of users & $15,30,60,90,120,150,180,210$ \\
\hline Users' Distribution & Random \\
\hline User speed & $3 \mathrm{Km} / \mathrm{h}$ \\
\hline Traffic type & Video \\
\hline Bit rate & $128,242,440$ kbps \\
\hline Maximum delay & 0.1 sec \\
\hline Buffer type & Infinite buffer \\
\hline Propagation model & Macro Urban channel realization \\
\hline
\end{tabular}

\section{SimUlation RESULTS}

The LTE-Sim-5 [11] was used in this paper after modifying it to support the first case of the CA. While the LTE-Sim-5 simulator is in the process of simulating a scenario with a pre-defined 
conditions, it takes into account both the signalling and data traffic. However, it only displays the data traffic in its traces. These data traffic traces are used to measure the QoS parameters, the system's average throughput, Packet Loss Rate (PLR), average packet delay, and fairness among users. These measurements are displayed in all the following figures by taking the number of users as its $\mathrm{X}$-axis factor and the QoS parameter as the $\mathrm{Y}$-axis factor.

\subsection{System's Average Throughput}

System's average throughput is defined as the amount of the total received packets for all users per second. The system's average throughput with and without the use of carrier aggregation for the three scheduling algorithms at different video bit-rates are displayed in the following figures "fig. 7, 8, and 9.

According to the obtained results that are displayed in figures "fig. 7, 8, and 9", increasing the number of users will increase the system's average throughput until it reaches its maximum value. This increase is due to transmitting more data from the eNodeB to the new added users. The maximum value of the system's average throughput differs based on the system's capabilities.

The use of CA will increase the bandwidth which will decrease the values of the spectral efficiency. This is defined in the literature as "the number of successfully transmitted bits normalized by the consumed resources in time and in bandwidth" [12]. This will prompt the eNodeB to transmit more data to users leading to higher system's average throughput which will in turn lead to increasing the spectral efficiency. This is shown in figures "fig. 7, and 8" in which the use of CA has slightly increased the system's average throughput even before reaching the maximum value of the system's average throughput in the case where the CA was not used.

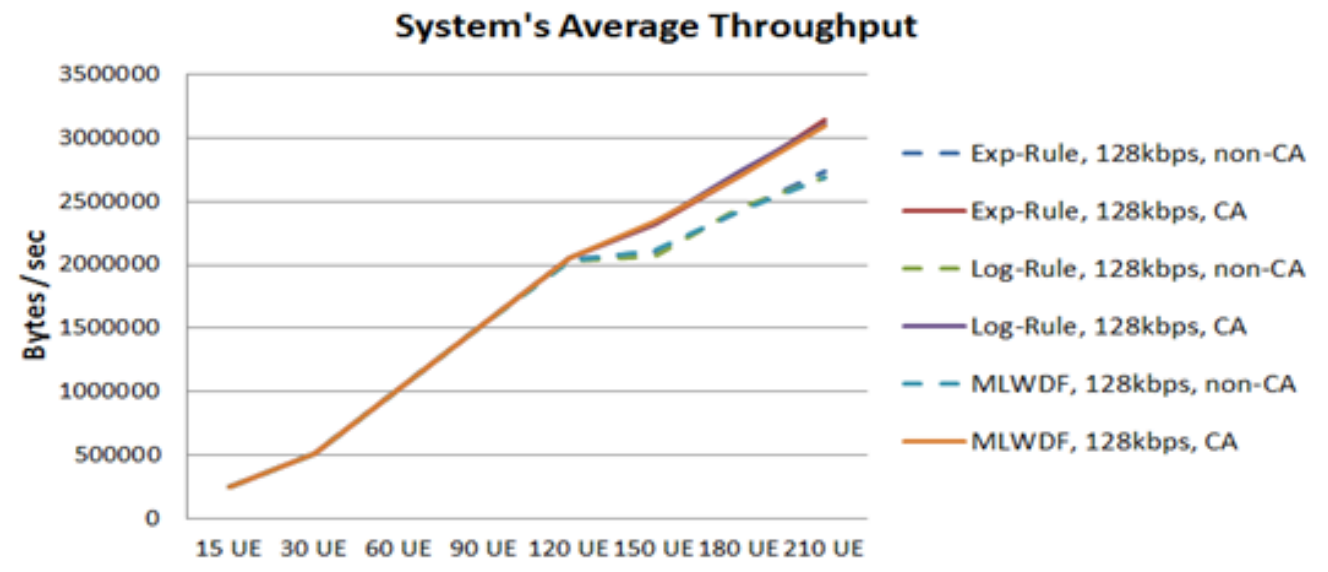

Figure 7. System's average throughput at video bit-rate $128 \mathrm{Kbps}$, with and without the use of CA

According to the obtained results that are displayed in figure 7, it is shown that at this scenario's conditions, the three algorithms showed similar performance in both cases. 


\section{System's Average Throughput}

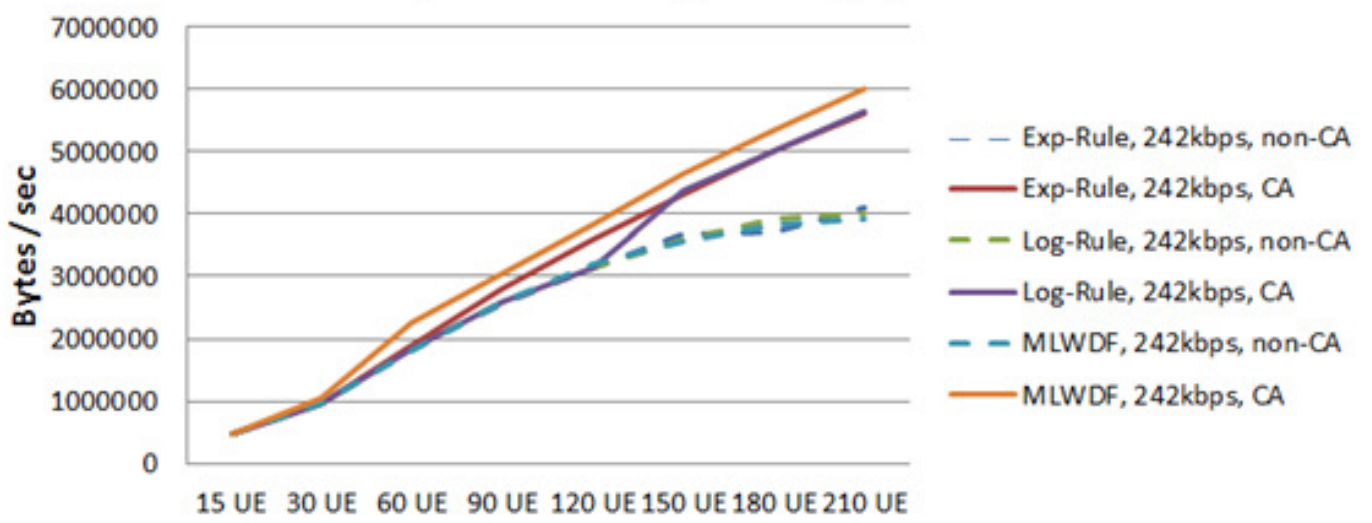

Figure 8 . System's average throughput at video bit-rate $242 \mathrm{Kbps}$, with and without the use of CA

According to the obtained results that are displayed in figure 8 , in both cases when the CA was and was not used, it is shown that the system's average throughput increased gradually by increasing the number of users. However, in the case in which the CA was not used and the increase of the system's average throughput started to reach its maximum value of between ( 4 and 4.5) MBps, this increase almost stopped. At this scenario's conditions, when the CA was not used, the three algorithms showed similar fluctuating performance. However, when the CA was used, the use of the MLWDF led to a slightly higher system's average throughput than the Exp-Rule, and the Log-Rule.

\section{System's Average Throughput}

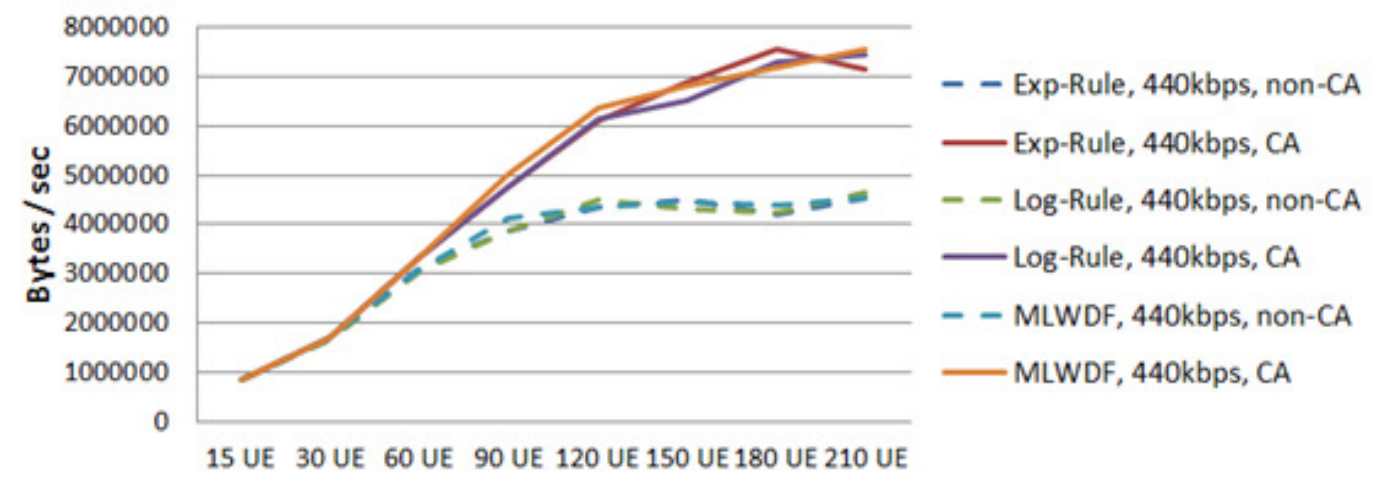

Figure 9. System's average throughput at video bit-rate $440 \mathrm{Kbps}$, with and without the use of CA

According to the obtained results that are displayed in figure 9, it is shown that the maximum value of the system's average throughput was almost doubled by the use of CA. At this scenario's conditions, the three algorithms showed a similar fluctuating performance in both cases.

\subsection{Packet Loss Rate (PLR)}

Packet Loss Rate (PLR) is measured by dividing the difference between the total transmitted and received packets for all users over the total transmitted packets. The greater the system's load the higher the value of the PLR. The system's load could be increased by increasing the number of users in the same cell, or by increasing the video bit-rates. The increase in the PLR values with the increase of the system's load is due to the increasing number of packets in the waiting queues that are competing for the same resource blocks. This will lead to a higher rate of dropped packets from these queues. Since both factors, the number of users and the video bit-rates, are proportionally related to increasing the PLR values, increasing one will limit the increase of the 
other in a limited resources system. For example, the significant increase of the PLR values started to take place when the number of users exceeded 120 users at a video bit-rate of $128 \mathrm{kbps}$, and when it exceeded 60 users at a video bit-rate of $242 \mathrm{kbps}$, and when it exceeded 30 users at a video bit-rate of $440 \mathrm{kbps}$. The increases of the PLR values with the increase of the number of users and the video bit-rates are shown in figures "fig. 10, 11, and 12". The results in these three figures, shows a decrease of the PLR values with the use of CA. This is because using the CA will increase the available resources and decrease the values of the spectral efficiency. This will prompt the eNodeB to allocate more resource blocks to the same user, which will reduce the amount of packets in the waiting queues, allowing more packets to be served and fewer packets to be dropped. This causes a decrease in the values of the PLR as shown in figures "fig. 10, 11, and 12 '.

The PLR with and without the use of carrier aggregation for the three scheduling algorithms at different video bit-rates are displayed in the following figures "fig. 10, 11, and 12".

\section{Packet Loss Rate}

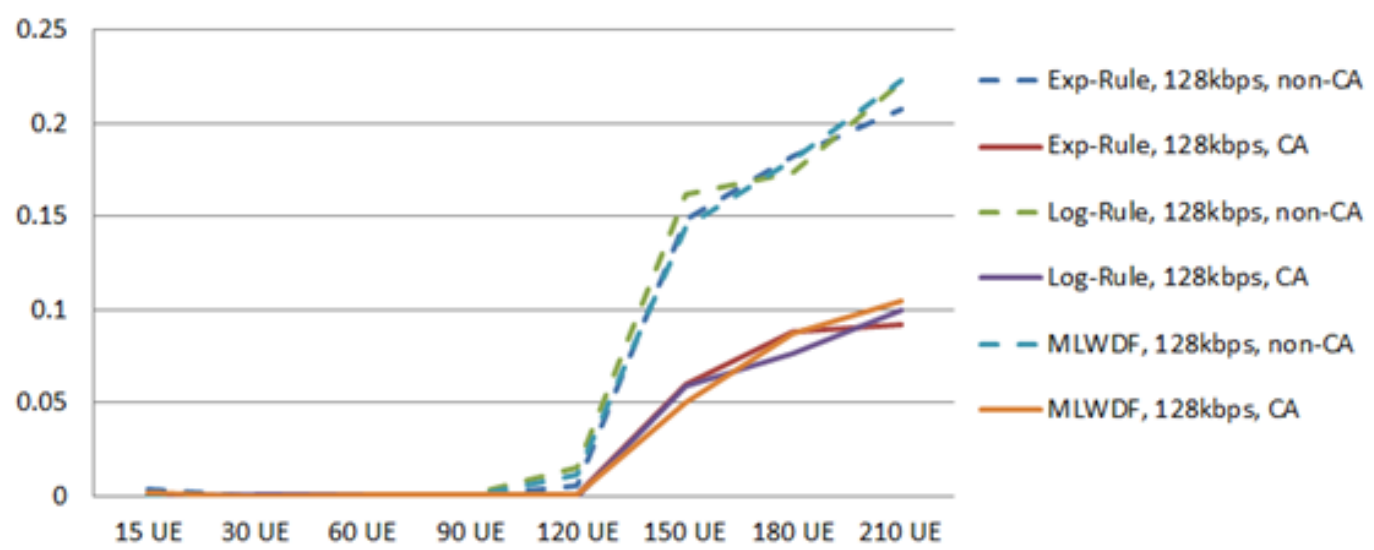

Figure 10. Packet Loss Rate (PLR) at video bit-rate $128 \mathrm{kbps}$, with and without the use of CA

\section{Packet Loss Rate}

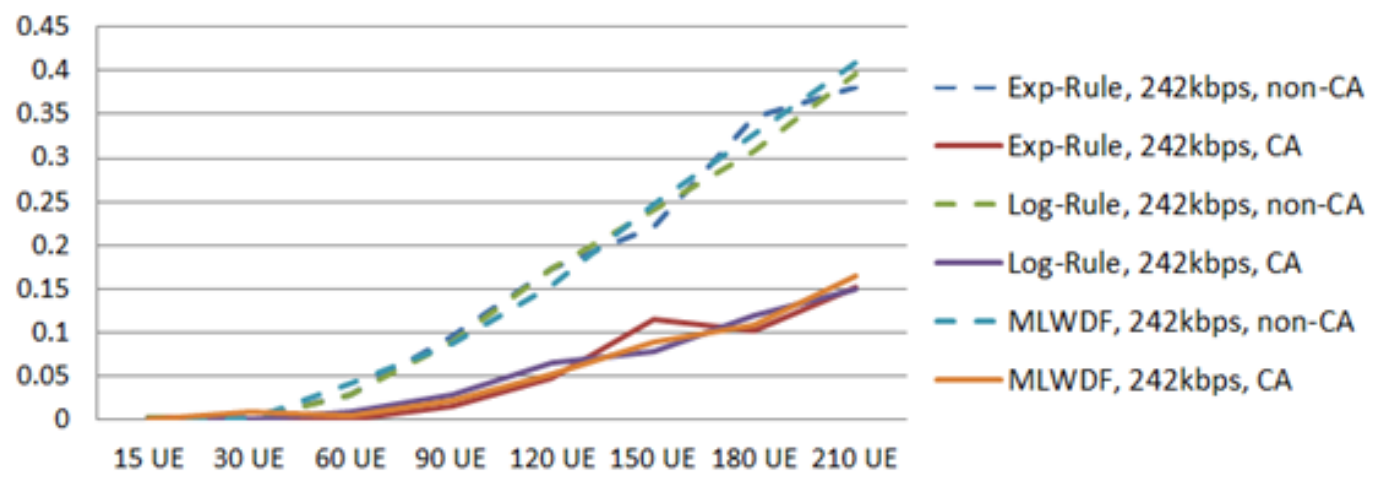

Figure 11. Packet Loss Rate (PLR) at video bit-rate 242kbps, with and without the use of CA

According to the obtained results that are displayed in figure 10, it is shown that the number of lost packet started to increase significantly when the number of users exceeded 120 UEs. However, when the CA was not used, this increase was twice what it was when the CA was used. At this scenario's conditions, the three algorithms showed similar fluctuating performance in both cases. 
According to the obtained results that are displayed in figure 11, the number of lost packets started to increase significantly after the number of users exceeded 60 UEs. However, when the CA was not used, the PLR was about two to three times what it was when the CA was used. At this scenario's conditions, the three algorithms showed similar fluctuating performance in both cases.

\section{Packet Loss Rate}

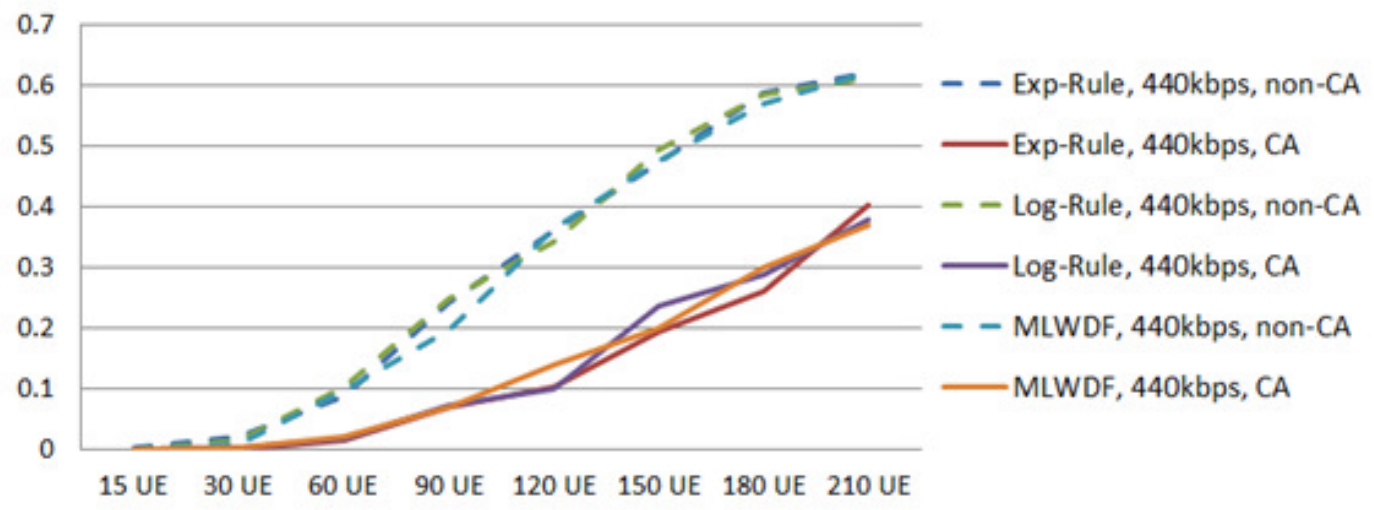

Figure 12. Packet Loss Rate (PLR) at video bit-rate 440kbps, with and without the use of CA

According to the obtained results that are displayed in figure 12, the number of lost packets started to increase critically after the number of users exceeded 30 UEs. However, when the CA was not used, the differences between the values of the PLR in both cases were on an average of twice what it was when the CA was used. At this scenario's conditions, the three algorithms showed similar fluctuating performance in both cases.

\subsection{Average Packet Delay}

The packet delay is the time that it takes a packet to travel from the source to its destination. It includes the propagation and waiting time of the packet. The Average Packet Delay is measured by dividing the sum of the total packet delays that were successfully received over the number of total packets. The use of the CA causes a significant beneficial reduction of the average packet delay. This is because it reduces the propagation time which is found by dividing the packet length by the link bandwidth. Also, it reduces the waiting time for the packets in the waiting queues at the eNodeB.

The average packet delay with and without the use of carrier aggregation for the three scheduling algorithms at different video bit-rates are displayed in the following figures "fig. 13, 14, and 15".

According to the obtained results that are displayed in figure 13, when the CA was not used, the average packet delay kept significantly increasing with the increase of the number of users into the the cell. While this was occurring, the PLR values remained almost negligible at this scenario's conditions, as seen in fig. 10. This led to a high queueing delay which peaked at 120 UE. However, when the number of users exceeded $120 \mathrm{UE}$, the waiting time for some of the packets in the waiting queues started to exceed the threshold defined as $0.1 \mathrm{sec}$, resulting in a higher rate of dropped packets. Consequently, the PLR values started to increase significantly. And since the average packet delay does not include in its calculations the dropped and lost packets, the values of it reduced significantly. This problem did not occur when the CA was used, because the available resources were almost doubled and the packets at the waiting queues were able to be served more quickly. At this scenario's conditions, the three algorithms showed similar fluctuating performance in both cases. 
Average Packet Delay

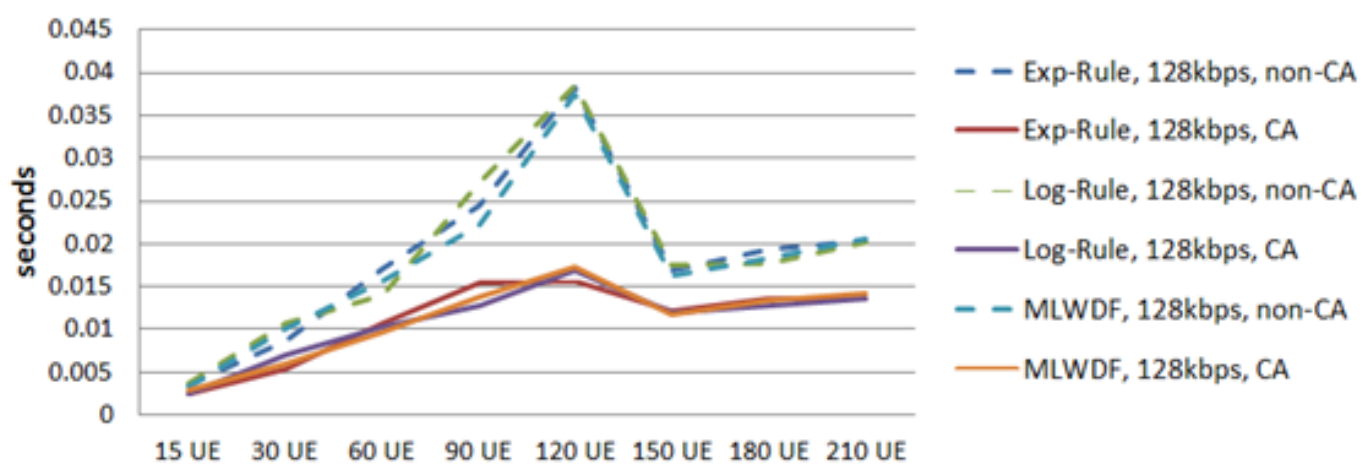

Figure 13. Average packet delay at video bit-rate $128 \mathrm{kbps}$, with and without the use of CA

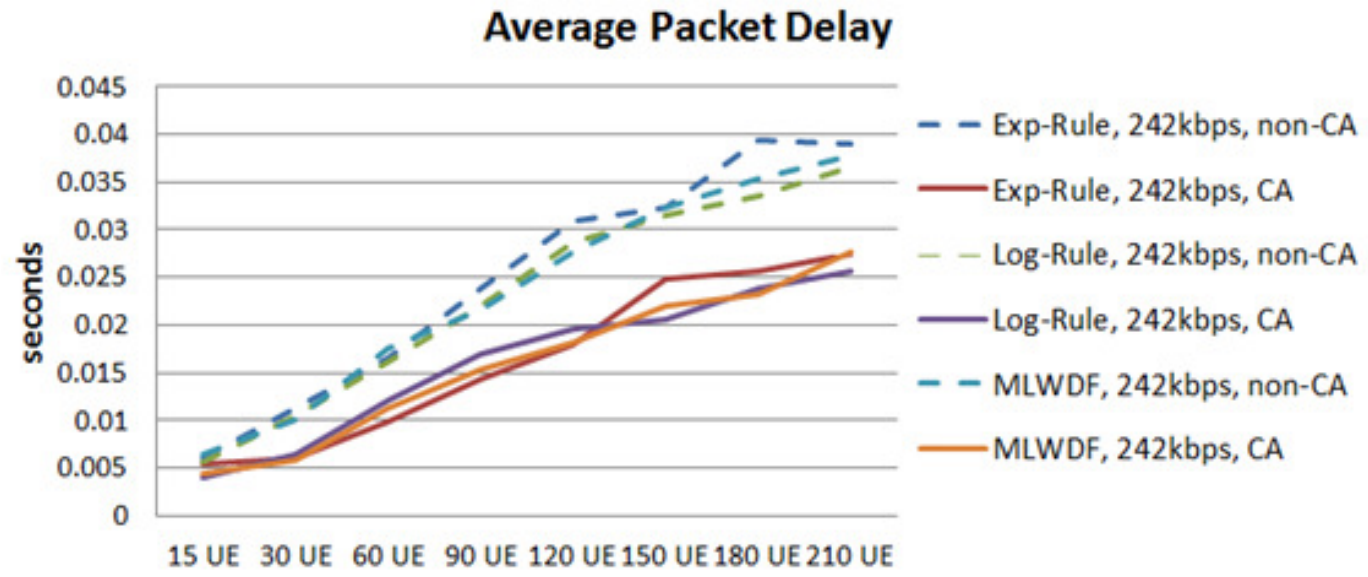

Figure 14. Average packet delay at video bit-rate $242 \mathrm{kbps}$, with and without the use of CA

\section{Average Packet Delay}

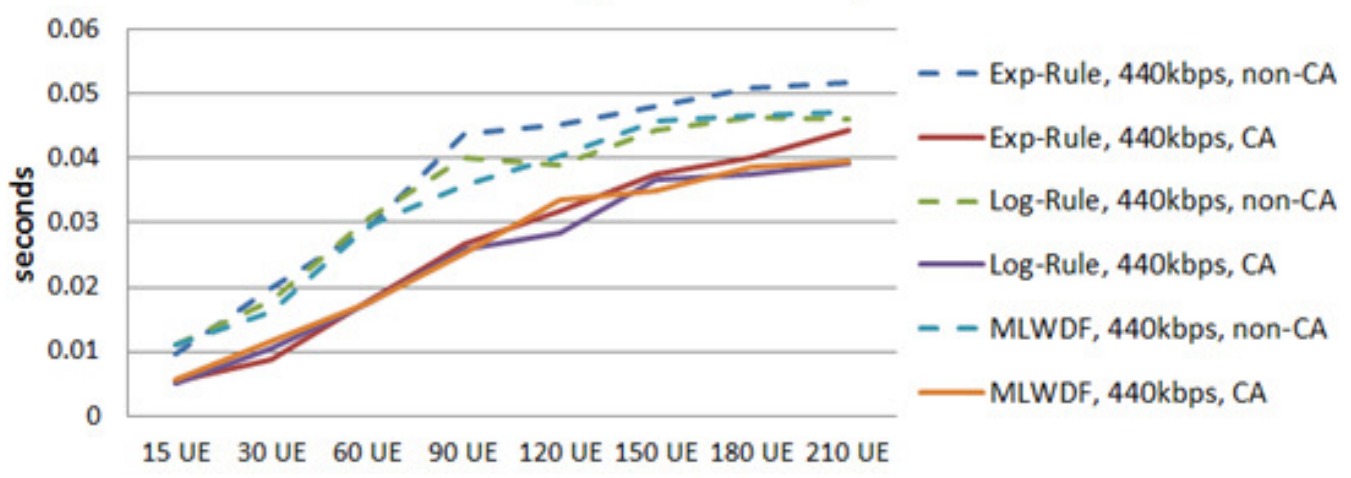

Figure 15. Average packet delay at video bit-rate 440kbps, with and without the use of CA

According to the obtained results that are displayed in figure 14, it is shown that the use of CA reduced the average packet delay to $60 \%$ of what it was when the CA was not used. The three algorithms showed similar fluctuating performance in both cases. However, the MLWDF results indicate that this algorithm has a more reliable performance in terms of increasing the average packet delay with increasing the number of users.

According to the obtained results that are displayed in figure 15, it is shown that the use of CA reduced the average packet delay to $50-60 \%$ of what it was when the CA was not used. This 
effect of the reduction continued until the number of UEs exceeded 90. After adding more users to the Macro-cell, this reduction started to decrease until the value of the average packet delay was almost $80 \%$ of what it was when the CA was not used. At this scenario's conditions, the three algorithms showed similar fluctuating performance in both cases with more performance stability to the MLWDF. The Exp-Rule had a slightly higher average packet delay than the other two algorithms.

\subsection{Fairness}

Jain's fairness index is used in this paper to determine if the scheduling algorithms are distributing fair portions of the spectrum to the users. It is measured by the following formula [13]:

$$
F=\frac{\left(\sum_{k=1}^{K} r_{k}\right)^{2}}{K \sum_{k=1}^{K} r_{k}}
$$

Where $r_{k}$ denotes the throughput of user $k$.

The Jain's fairness index for the three scheduling algorithms at different video bit-rates, with and without the use of carrier aggregation, are displayed in the following figures "fig. 16, 17, and 18".

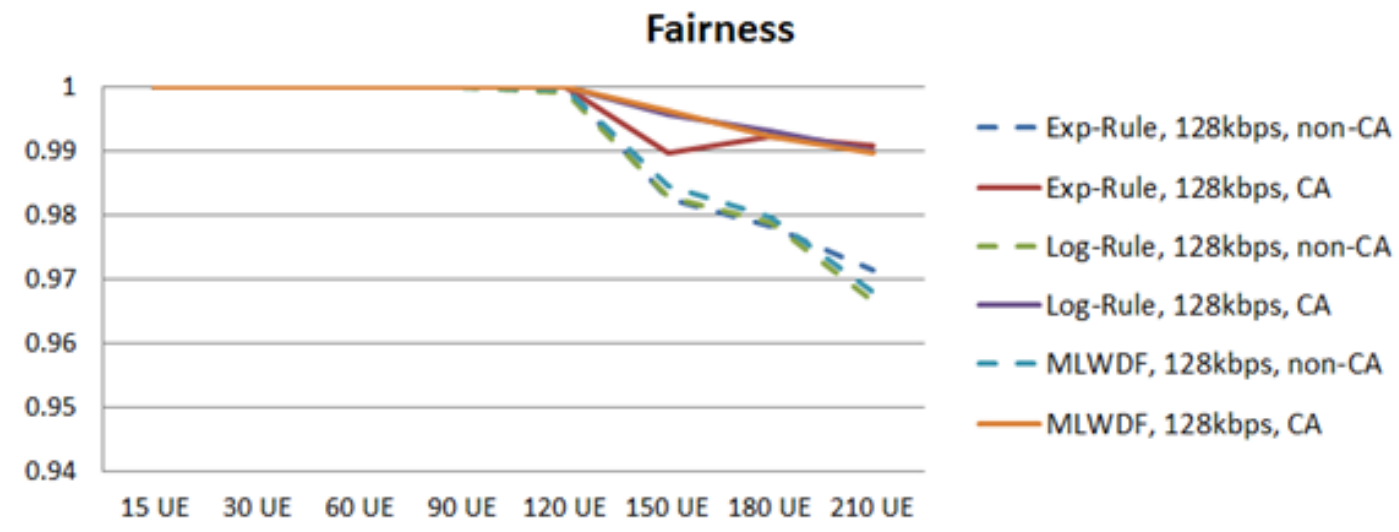

Figure 16. Fairness index for the three algorithms at video bit-rate $128 \mathrm{kbps}$, with and without the use of CA

According to the obtained results that are displayed in figure 16, at this scenario's conditions, the three algorithms showed similar fluctuating fairness in both cases. An excellent fairness took place among users for the three algorithms when the CA was used. However, when the CA was not used, the fairness indicator dropped slightly after the number of users exceeded 120 UEs.

According to the obtained results that are displayed in figure 17, at this scenario's conditions, the three algorithms showed similar fluctuating fairness in both cases. An excellent fairness was forced among users for the three algorithms when the CA was used. However, when the CA was not used, the fairness indicator dropped 5-20\% from what it was when the CA was used, this drop started to take place after the number of users exceeded 120 UEs.

According to the obtained results that are displayed in figure 18, at this scenario's conditions, the three algorithms showed similar fluctuating fairness in both cases. When the CA was used, and when the number of users started to exceed $90 \mathrm{UE}$, the fairness indicator started to drop until it reached a value of 0.8 when the number of users was 210 UE. When the CA was not used the drop of the fairness indicator was more significant, it reached to a value of 0.6 when the number of users was $210 \mathrm{UE}$. 


\section{Fairness}

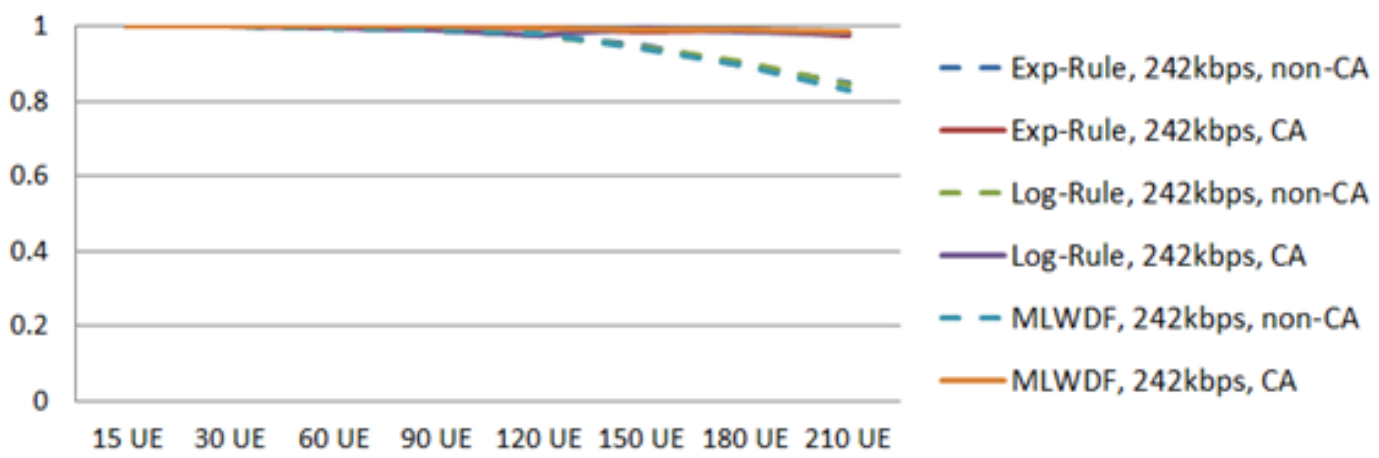

Figure 17. Fairness index for the three algorithms at video bit-rate $242 \mathrm{kbps}$, with and without the use of CA

Fairness

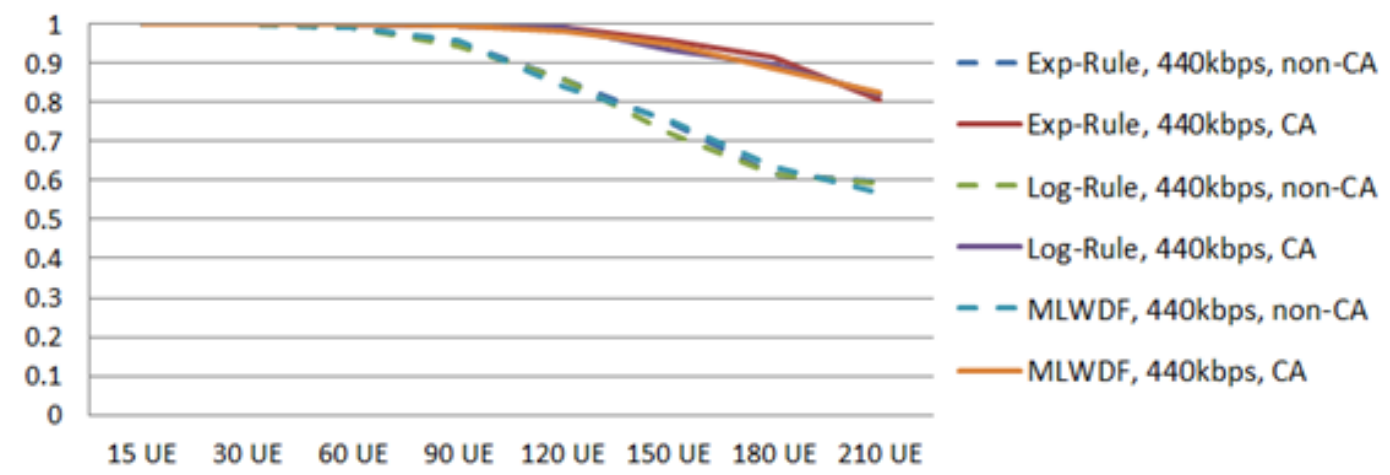

Figure 18. Fairness index for the three algorithms at video bit-rate $440 \mathrm{kbps}$, with and without the use of CA

\section{CONCLUSION}

This paper has provided a comparative study on three Channel-aware/QoS-aware scheduling algorithms over LTE/LTE-A for video-applications. The comparison aimed to study the behaviour of the selected algorithms when the network load reaches the capacity limitations of the LTE macro-cell and to compare it when the network is supported by the CA feature to operate as LTE-A. In addition, there was a comparison among the scheduling algorithms in both cases. The evaluation process was based on simulating different scenarios by varying the number of users, the video bit-rates, and the system's bandwidth. The LTE-Sim-5 was used in the simulation process both with and without modifications. The QoS performance evaluation was in terms of the QoS parameters, the system's average throughput, Packet Loss Rate (PLR), average packet delay, and fairness among users. Simulation results show that the system's average throughput was significantly improved by the use of CA. The capacity limitation boundaries were also doubled. The use of the MLWDF slightly improved the system's average throughput while the CA was used. The PLR was significantly reduced almost $50 \%$ by the use of CA at a video bit-rate of $128 \mathrm{kbps}$, by $50-70 \%$ at a video bit-rate of $242 \mathrm{kbps}$, and by $40-60 \%$ at a video bit-rate of $440 \mathrm{kbps}$. The average packet delay was reduced by the use of CA at a video bit-rate of $128 \mathrm{kbps}$, and at a video bit-rate of $242 \mathrm{Kbps}$ by $30-40 \%$, and at a video bit-rate of $440 \mathrm{kbps}$ by $20-30 \%$. The MLWDF showed more performance stability in terms of increasing the average packet delay with increasing the number of users. The Exp-Rule had a slightly higher delay than the LOG-rule and the MLWDF. The fairness indicator was improved with the use of CA by a factor of $10-20 \%$. 
These results show that the use of CA is worth being investigated by researchers, implemented by the manufacturers, and deployed by the service providers.

\section{APPENDix A: LiST OF ACRONYMS}

$\begin{array}{ll}\text { 3GPP } & \text { Third Generation Partnership Project } \\ \text { AMC } & \text { Adaptive Modulation and Coding } \\ \text { AuC } & \text { Authentication Center } \\ \text { CA } & \text { Carrier Aggregation } \\ \text { CC } & \text { Component Carrier } \\ \text { CQI } & \text { Channel Quality Indicator } \\ \text { DCI } & \text { Downlink Control Information } \\ \text { eNodeB } & \text { Evolved NodeB } \\ \text { EPC } & \text { Evolved Packet Core } \\ \text { Exp-Rule } & \text { Exponential Rule } \\ \text { FDD } & \text { Frequency Division Duplex } \\ \text { GBR } & \text { Guaranteed Bit Rate } \\ \text { GPRS } & \text { General Packet Radio Service } \\ \text { HOL } & \text { Head of Line } \\ \text { HSS } & \text { Exponential Rule } \\ \text { LTE } & \text { Long Term Evolution } \\ \text { LTE-A } & \text { LTE-Advanced } \\ \text { Log-Rule } & \text { Logarithmic Rule } \\ \text { LWDF } & \text { Largest Weighted Delay First } \\ \text { MAC } & \text { Medium Access Control } \\ \text { MCS } & \text { Modulation and Coding Scheme } \\ \text { MLWDF } & \text { Modified Largest Weighted Delay First } \\ \text { MME } & \text { Mobility Management Entity } \\ \text { OFDM } & \text { Orthogonal Frequency Division Multiplexing } \\ \text { PCEF } & \text { Policy Control Enforcement Function } \\ \text { PCRF } & \text { Policy Control and Charging Rules Function } \\ \text { PDCCH } & \text { Physical Downlink Control Channel } \\ \text { PDSCH } & \text { Physical Downlink Shared Channel } \\ \text { PDCP } & \text { Packet Data Control Protocol } \\ \text { PDNs } & \text { Packet Data Networks } \\ \text { PF } & \text { Proportional Fairness } \\ \text { PLR } & \text { Packet Loss Rate } \\ \text { PUSCH } & \text { Physical Uplink Control Channel } \\ \text { P-GW } & \text { PDN-Gateway } \\ \text { QAM } & \text { Quadrature Amplitude Modulation } \\ \text { QoS } & \text { Quality of Service } \\ \text { QPSK } & \text { Quadrature Phase-Shift Keying } \\ \text { R8/9 } & \text { Release 8/9 } \\ \text { R10/11 } & \text { Release 10/11 } \\ \text { RAN } & \text { Radio Access Network } \\ \text { RB } & \text { Resource Block } \\ \text { RLC } & \text { Radio Link Control } \\ \text { RRC } & \text { Radio Resource Control } \\ \text { S-GW } & \text { Serving Gateway } \\ \text { TFTs } & \text { Traffic Flow Templates } \\ \text { TTI } & \text { Transmission Time Interval } \\ \text { UE } & \text { User Equipment } \\ \text { UMTS } & \text { Universal Mobile Telecommunication Systems } \\ & \end{array}$


VoIP Voice over IP

Wi-Fi wireless fidelity

WiMAX Worldwide Interoperability for Microwave Access

\section{REFERENCES}

[1] “3gpp, “lte.” [online]. available:.” http://www.3gpp.org/technologieskeywords-acronyms/98-lte

[2] F. Capozzi, G. Piro, L. A. Grieco, G. Boggia, and P. Camarda, "Downlink packet scheduling in lte cellular networks: Key design issues and a survey," Communications Surveys \& Tutorials, IEEE, vol. 15, no. 2, pp. 678-700, 2013.

[3] “3gpp, "carrier aggregation explained.” [online]. Available:." http://www.3gpp.org/technologies/keywords-acronyms/101-carrier-aggregation-explained

[4] H. Holma and A. Toskala, LTE for UMTS-OFDMA and SC-FDMA based radio access. John Wiley \& Sons, 2009.

[5] S. P. E. Dahlman and J. Skold, 4G LTE/LTE-Advanced for Mobile Broadband. Elseiver, 2011.

[6] "Anritsu, "lte resource guide." [online]. available:." http://www.3gpp.org/technologies/keywords-acronyms/98-lte

[7] A. T. H. Holma, LTE Advanced: 3GPP Solution for IMT-Advanced. Wiley, 2012.

[8] "Anritsu, understanding lte-advanced carrier aggregation. [online]. Available:." http://downloadfile.anritsu.com/RefFiles/en-GB/Promotions/Understanding-Carrier-Aggregationweb.pdf

[9] B. Liu, H. Tian, and L. Xu, "An efficient downlink packet scheduling algorithm for real time traffics in lte systems," in Consumer communications and networking conference (CCNC), 2013 IEEE, pp. 364-369, IEEE, 2013.

[10] M. Iturralde, T. Ali Yahiya, A. Wei, and A.-L. Beylot, "Performance study of multimedia services using virtual token mechanism for resource allocation in lte networks," in Vehicular Technology Conference (VTC Fall), 2011 IEEE, pp. 1-5, IEEE, 2011.

[11] G. Piro, L. A. Grieco, G. Boggia, F. Capozzi, and P. Camarda, "Simulating lte cellular systems: an open-source framework," Vehicular Technology, IEEE Transactions on, vol. 60, no. 2, pp. 498-513, 2011.

[12] Y. Chen and B. Walke, "Analysis of cell spectral efficiency in 3gpp lte systems," in Personal Indoor and Mobile Radio Communications (PIMRC), 2013 IEEE 24th International Symposium on, pp. 1799-1804, IEEE, 2013.

[13] C. Bae and D.-H. Cho, "Fairness-aware adaptive resource allocation scheme in multihop ofdma systems," Communications Letters, IEEE, vol. 11, no. 2, pp. 134-136, 2007. 
